\title{
Modulation of gyrosynchrotron emission in solar and stellar flares by slow magnetoacoustic oscillations
}

\author{
V. M. Nakariakov ${ }^{1}$ and V. F. Melnikov ${ }^{2}$ \\ 1 Physics Department, University of Warwick, Coventry CV4 7AL, UK \\ e-mail: valery@astro.warwick.ac.uk \\ 2 Radiophysical Research Institute (NIRFI), Nizhny Novgorod 603950, Russia \\ e-mail: meln@nirfi.sci-nnov.ru
}

Received 29 July 2005 / Accepted 20 September 2005

\section{ABSTRACT}

\begin{abstract}
Gyrosynchrotron emission generated by non-thermal electrons in solar and stellar coronal flares can be efficiently modulated by slow magnetoacoustic oscillations in the flaring loops. The modulation mechanism is based upon perturbation of the efficiency in the Razin suppression of optically thin gyrosynchrotron emission. Modulation of the emission is in anti-phase with the density perturbation in the slow wave. The observed emission modulation depth can be up to an order of magnitude higher than the slow wave amplitude. This effect is more pronounced at lower frequencies. Observations with spatial resolution, together with analysis of the modulation frequency, are shown to be sufficient for providing the information needed to identify the mode.
\end{abstract}

Key words. Sun: flares - Sun: oscillations - Sun: corona - stars: flare - stars: oscillations - stars: coronae

\section{Introduction}

Oscillations with the periods from one second to tens of minutes in the radio, X-ray, and EUV emission coming from the solar corona have been observed for several decades (see Aschwanden 2004; Nakariakov \& Verwichte 2005, for recent reviews). The high spatial resolution of modern solar coronal observational tools allows identification of specific modes of the coronal oscillation in terms of magnetohydrodynamic (MHD) wave theory. Recent progress in the observational detection and study of coronal oscillations and waves reinforces wave-based theories for coronal heating and provides us with the basis for a new method of determining coronal physical parameters - MHD coronal seismology.

The most frequently observed in solar coronal structures MHD mode is the slow magnetoacoustic wave. This mode has been identified as periodic EUV emission disturbances propagating along polar plumes (DeForest \& Gurman 1998; Ofman et al. 1999) and along long loops (Berghmans \& Clette 1998), with typical periods ranging from two to fifteen minutes. The typical relative amplitudes of the EUV propagating disturbances are usually weaker than $10-15 \%$, and the projected propagation speeds range from 30 to $160 \mathrm{~km} \mathrm{~s}^{-1}$ (see, e.g., De Moortel et al. 2002). Theoretical models of this phenomenon, which interpret it in terms of slow magnetoacoustic waves, were developed for plumes by Ofman et al. (1999) and by Nakariakov et al. (2000) for loops.
Recently, a standing slow magnetoacoustic mode was detected as the Doppler shift and emission intensity variations of EUV emission (Wang et al. 2002, 2003). The oscillation periods were found in the range of 7 to $30 \mathrm{~min}$ with the mean at $18 \mathrm{~min}$, and the oscillation amplitude is typically about $10-20 \%$, in some cases reaching $50 \%$ of the background. The line-of-sight (LOS) velocity variations estimated from the Doppler shift were found to be shifted by the quarter period with respect to the intensity variations (Wang et al. 2003). Such behaviour is consistent with the theoretical model of this phenomenon suggested by Ofman \& Wang (2002). In this simple model, the slow magnetoacoustic oscillation of a coronal loop was modelled as a global standing mode of a one-dimensional acoustic resonator formed by unpenetrated footpoints and filled by a uniform plasma. This study was developed by Mendoza-Briceño et al. (2004), who incorporate stratification effects in the model. Their results confirm the applicability of Ofman \& Wang's model, as the discrepancy between the values of the oscillation period calculated in the stratified and in the non-stratified models was only a few percent. The practical formula for estimating the period of the global slow mode is

$P / \mathrm{s} \approx 13 \times(L / \mathrm{Mm}) / \sqrt{(T / \mathrm{MK})}$.

Modelling response of a coronal loop to an impulsive deposition of energy, Nakariakov et al. (2004) established that standing slow magnetoacoustic oscillations with typical 
density perturbation amplitudes less than 10-20\% are an intrinsic feature of this process. It was found that, in the case of the energy deposition locatation near the loop top, the preferentially excited mode is the second harmonics. The period of this mode is approximately two times shorter than the period of the global mode given by Eq. (1). For the characteristic lengths and temperatures of flaring loops, the typical periods range from tens of seconds to several minutes. Both numerical simulations of Mendoza-Briceño et al. (2004) and of Nakariakov et al. (2004) show that the slow magnetoacoustic oscillation is almost isothermal because of the high thermal conduction in the plasma. The structure of the global slow magnetoacoustic mode and of its second spatial harmonics is shown in Fig. 1; the global mode has a node of the density perturbation at the loop top, while the second harmonics has a maximum of the density perturbation there and two nodes at the loop legs. This property, together with the difference in oscillation periods, can be used to identify the mode.

Discovery of the slow magnetoacoustic mode in the EUV coronal dynamics stimulates an interest in using this mode in interpreting the coronal oscillations observed in other bands. In particular, the periodicities range from a few tens of seconds to tens of minutes are detected in the microwave band. For example, Baranov \& Tsvetkov (1994) found $22 \mathrm{~s}$, $30 \mathrm{~s}$, and $34 \mathrm{~s}$ periodicities in the gyrosynchrotron emission at $8.5-15 \mathrm{GHz}$ with the RT-22 at Crimea. Khodachenko et al. (2005) detected oscillations with the period of $20 \mathrm{~s}$ in the flaregenerated $37 \mathrm{GHz}$ microwave radiation recorded at Metsähovi. In these observations, there are also oscillations with much longer periods, from 5 to $15 \mathrm{~min}$, that are clearly seen in the flaring light curves. Periodicities in this range are also detected in the microwave emission generated in stellar flares, e.g. oscillations with the period of $56 \mathrm{~s}$ were found by Gary et al. (1982) on L726-8 A at $4.9 \mathrm{GHz}$ with VLA.

In flare events, microwaves can be efficiently produced by non-thermal electrons through the gyrosynchrotron mechanism. The aim of this paper is to investigate the possibility of modulating of the gyrosynchrotron emission intensity and hence the microwave radiation coming from flaring loops by long-period acoustic waves.

\section{Modulation of the gyrosynchrotron emission spectrum}

Gyrosynchrotron emission is caused by the interaction of nonthermal particles, in particular the electrons accelerated in solar flares, with a background magnetic field. The efficiency of the emission is affected by the parameters of the non-thermal electrons. Their energy distribution is usually modelled by a power law $N(E)=K E^{-\delta}$, where $K$ is the number density of electrons in an unity energy interval, and $\delta$ is the power law index that is usually in the range $2<\delta<7$. Also, the emission efficiency depends upon the source plasma (the magnetic field $B$ and the density $n_{\mathrm{e}}$ ) and upon the observation conditions (the angle $\theta$ between the LOS and the local magnetic field and the volume of the observed emitting source, which includes the column depth of the source). The gyrosynchrotron spectrum has a well-defined peak at frequency $f_{\text {peak }}$. At the frequencies

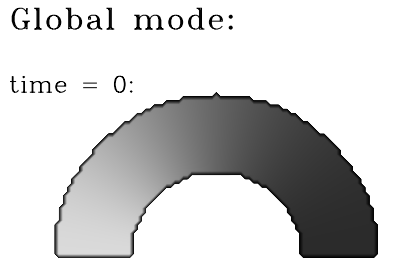

\section{Second harmonics:}
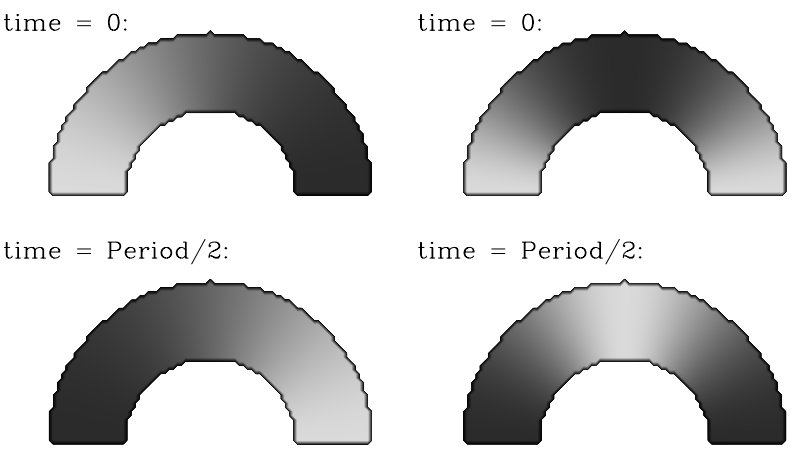

time $=$ Period $/ 2$ :

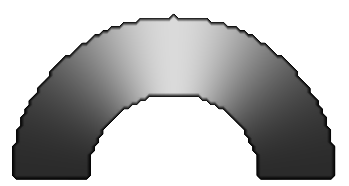

Fig. 1. The structure of the density perturbations along a loop in the global slow magnetoacoustic mode (left column) and in the second harmonics (right column) at two different instants of time. The brightness of the filling corresponds to the plasma density.

higher that the peak frequency, $f>f_{\text {peak }}$, the source of emission is optically thin, and the intensity is defined mostly by the electron energy distribution and the magnetic field strength and direction.

In flaring loops that contain a low density plasma the emission intensity at the frequencies $f \leq f_{\text {peak }}$ is usually defined by the effect of self-absorption. In this case, the variation of the plasma density has no effect on the emission in the all microwave frequency range.

However, the situation changes drastically in the case of sufficiently high plasma density in a flaring loop. At higher frequencies, $f \gg f_{\text {peak }}$, the source is optically thin for the microwaves and the emission efficiency is still independent of the plasma density. But at lower frequencies, below or comparable to a so-called Razin frequency, the gyrosynchrotron emission can be subject to Razin suppression (Razin 1960). The Razin frequency is defined by the expression

$f_{\mathrm{R}}=2 f_{\mathrm{p}}^{2} / 3 f_{B} \approx 20 \times n_{\mathrm{e}} / B$,

where $f_{\mathrm{p}}$ - is plasma frequency and $f_{B}$ is gyrofrequency. In the estimation, the density is in $\mathrm{cm}^{-3}$ and the field is in $\mathrm{G}$, giving the Razin frequency in Hz. The effect of the ratio $n_{\mathrm{e}} / B$ on the emission intensity at the lower frequencies $f \ll f_{\mathrm{R}}$ may can be seen well in the synchrotron approach (Razin 1960; Goundyrev \& Razin 1995):

$I_{f} \propto\left(\frac{2 f}{3 f_{\mathrm{R}}}\right)^{1-\delta} \exp \left(-\frac{3^{2 / 3} f_{\mathrm{R}}}{2 f}\right)$.

Under a constant magnetic field, any increase/decrease of the plasma density should lead to a subsequent decrease/increase in the emission intensity. Consequently, modulation of the plasma density by a compressible wave, e.g. a slow magnetoacoustic wave, is projected onto the efficiency of Razin suppression of the gyrosynchrotron emission, making the slow magnetoacoustic waves observable at microwaves. Recent observations performed with high spatial resolution (Melnikov et al. 2005a), as well as with high spectral resolution (Fleishman et al. 2004; Melnikov et al. 2005b), provide us with ample evidence of the presence of the plasma density sufficient for strong Razin suppression in a number of flaring loops. 


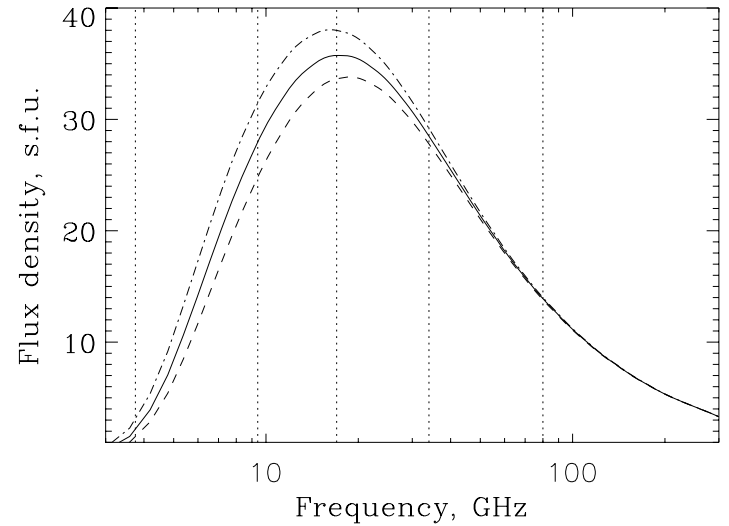

Fig. 2. Microwave gyrosynchrotron spectra coming from a segment of a flaring loop with the magnetic field of $100 \mathrm{G}$ and the plasma concentration of $4 \times 10^{10} \mathrm{~cm}^{-3}$, observed from an angle $45^{\circ}$ to the local magnetic field, modulated by a slow magnetoacoustic oscillation. The solid curve corresponds to the unperturbed plasma density; the dashed curves correspond to the 5\%-enhancement and dash-dotted ones to a decrease in the plasma density by a slow magnetoacoustic wave. The vertical dotted lines correspond to the frequencies of $3.75,9.4,17,34$, and $80 \mathrm{GHz}$.

In the following, we restrict our attention to the case of parallel propagation of the slow magnetoacoustic waves, when they degenerate into the pure acoustic waves and do not perturb the magnetic field at all. Also, in the low- $\beta$ plasma typical of coronal loops, slow magnetoacoustic waves do not produce any noticeable perturbations of the loop cross-section, as the change in the gas pressure produced by them is negligible in comparison with the magnetic pressure. Consequently, the only possible contribution of the slow magnetoacoustic waves to modulation of the gyrosynchrotron emission is through the density perturbations.

Typical microwave spectra of the gyrosynchrotron emission coming from a flaring loop, calculated according to the exact formalism that includes both the self-absorption and Razin suppression, are shown in Fig. 2. A detailed description of the formalism and the numerical method used can be found in Ramaty (1969) and Fleishman \& Melnikov (2003). Here, for simplicity, we assume that the non-thermal electrons have a power-law energy distribution with an isotropic pitch angle distribution. The other parameters used in the simulation are: the magnetic field $B=100 \mathrm{G}$, the plasma concentration is $n_{\mathrm{e}}=4 \times 10^{10} \mathrm{~cm}^{-3}$, the non-thermal electron power law in$\operatorname{dex} \delta=3$, and the LOS angle $\theta=45^{\circ}$. The corresponding values of the characteristic frequencies are $f_{\mathrm{p}} \approx 1.8 \mathrm{GHz}$, $f_{B} \approx 280 \mathrm{MHz}$, and $f_{\mathrm{R}} \approx 7.7 \mathrm{GHz}$. The plasma density is modulated by a low frequency slow magnetoacoustic wave of the amplitude $5 \%$. Figure 2 confirms the expected result (see Eq. (3)) that the efficiency of the gyrosynchrotron emission decreases with the increase in the plasma density. This effect is more pronounced at the frequencies near to and lower than the spectrum peak frequency. The perturbations of the emission are in anti-phase with the perturbations of the plasma density in the slow magnetoacoustic wave. Figure 3 demonstrates the modulation of the emission at different frequencies, including $17 \mathrm{GHz}$ and $34 \mathrm{GHz}$ - the observation frequencies of the
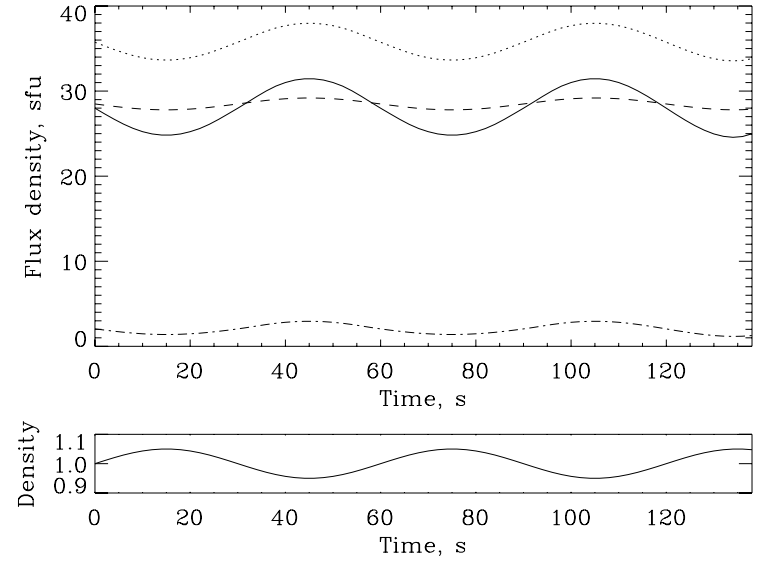

Fig. 3. Upper panel: modulation of the microwave emission produced by a slow magnetoacoustic wave with the period of one minute and the amplitude of 5\% at specific microwave frequencies. All parameters are the same as in Fig. 2. The dash-dotted curve corresponds to $3.75 \mathrm{GHz}$, the solid to $9.4 \mathrm{GHz}$, the dotted to $17 \mathrm{GHz}$, and the dashed to $34 \mathrm{GHz}$. The modulating slow magnetoacoustic wave with the $5 \%$ amplitude is shown in the bottom panel.

Nobeyama Radioheliograph (NoRH). At the frequencies near and below the spectral peak, the modulation depth of the emitting signal can be significantly larger than the amplitude of the modulating wave, while the effect is practically absent at higher frequencies.

Emission $I_{f}$ at different frequencies $f$ is affected by the perturbations of the plasma density in a slow magnetoacoustic wave with different efficiency, which can be characterised by the modulation factor,

$\Delta=\frac{I_{f}^{\max }-I_{f}^{\min }}{I_{f}^{\max }+I_{f}^{\min }} \frac{1}{A}$,

where $I_{f}^{\max }$ and $I_{f}^{\min }$ are the maximum and the minimum values of the modulated emission $I_{f}$, and $A$ is the wave relative amplitude. In other words, the modulation factor is a ratio of the relative amplitude of the perturbation, observed at frequency $f$ and the actual amplitude $A$ of the modulating wave. Figure 4 gives the dependencies of the modulation factor upon different parameters. For an optimal combination of the parameters, the modulation factor can be up to 10 , meaning that the observed modulation depth of the microwave emission can be an order of magnitude higher than the amplitude of the slow magnetoacoustic wave. Normally, the high modulation factor can be achieved in the lower part of the microwave spectrum. The modulation factor increases with the plasma density, with the decrease in the magnetic field, and with the decrease in the LOS angle to the local magnetic field. The modulation factor is practically independent of the power law spectral index of the non-thermal electrons that cause the emission. We would like to stress that the modulation factor $\Delta$ is a relative characteristic of the modulation depth, which demonstrates the amplification or attenuation of the actual signal. Note that in the case of a strong magnetic field ( $B=400 \mathrm{G}$, see the dash-dotted line in Fig. 4), the effect of the amplification at low frequencies disappears due 

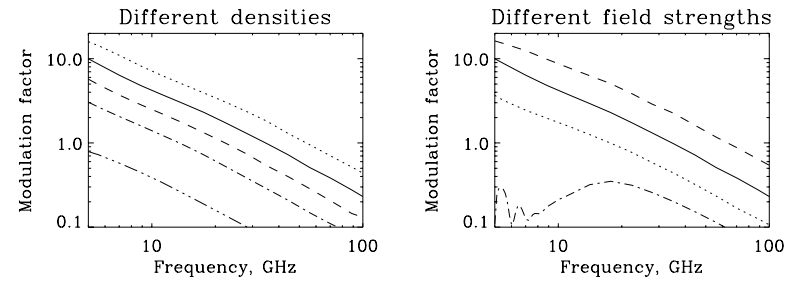

Different $\delta$
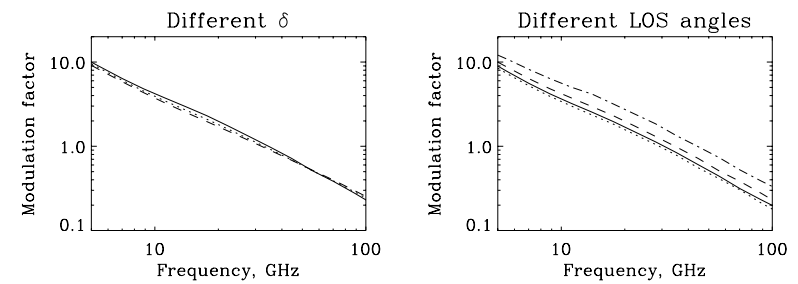

Fig. 4. Dependencies of the modulation factor (the ratio of the observed amplitude to the actual amplitude) upon parameters. The solid curve in all panels is the same and corresponds to the plasma with the magnetic field $B=100 \mathrm{G}$, the density $n_{\mathrm{e}}=8 \times 10^{10} \mathrm{~cm}^{-3}$, the non-thermal electron spectral index $\delta=3$, and the observation angle $\theta=66^{\circ}$. Other curves show the modification of this curve by varying parameters. The upper left panel: the dotted curve corresponds to $n_{\mathrm{e}}=15 \times 10^{10} \mathrm{~cm}^{-3}$, the dashed to $5 \times 10^{10} \mathrm{~cm}^{-3}$, the dash-dotted curve to $3 \times 10^{10} \mathrm{~cm}^{-3}$, and the dot-dot-dashed curve to $10^{10} \mathrm{~cm}^{-3}$. The upper right panel: the dashed line corresponds to $B=50 \mathrm{G}$, the dotted to $B=200 \mathrm{G}$, and the dash-dotted to $B=400 \mathrm{G}$. The lower left panel: the dashed curve corresponds to $\delta=5$ and the dotted to $\delta=4$. The lower right panel: the dotted curve corresponds to $\theta=78^{\circ}$, the dashed to $\theta=53^{\circ}$, and the dash-dotted to $\theta=37^{\circ}$.

to weakening of the Razin suppression and strengthening of the self-absorption $(\tau>1)$.

\section{Spatially resolved observations}

The resolution of the spatial structure of a loop oscillation is a crucial condition of correct mode identification. Global and second harmonic slow magnetoacoustic modes of a loop have wavelengths that are larger than the loop radius and consequently can be resolved easily with modern radioheliographs, e.g. with NoRH as was done recently for global sausage modes (Nakariakov et al. 2003; Melnikov et al. 2005a) and possibly kink modes (Asai et al. 2001). In the following we consider possible manifestation of slow modes in observations with sufficiently high spatial resolution.

Suppose that a flaring loop is observed from above and that the LOS is, for simplicity, parallel to the loop plane and has a certain angle $\phi$ to the horizon (see Fig. 5). Also, we assume that the loop is sufficiently hot and compact, allowing us to neglect the effect of plasma stratification, and that the nonthermal electrons are uniformly distributed in the loop. In the optically thin regime, different segments of the loop have different observed column depths, because of the loop curvature. In the microwave band, the change of the magnetic field vector will also contribute to the spatial structure of the observed emission. Under the above assumptions, the microwave emission coming from the loop to the radioheliograph will have a certain profile along the observed loop axis. A typical profile resembling St Exupery's "elephant-in-a-python" is shown by

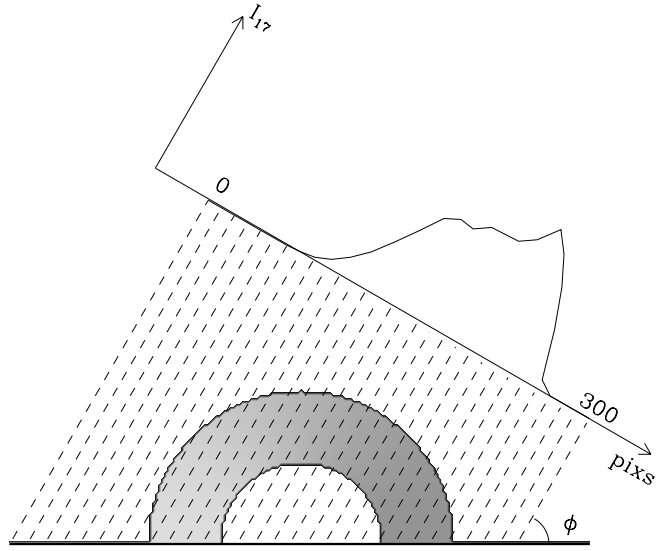

Fig. 5. A sketch of a possible spatially resolving observation of a coronal loop experiencing a slow magnetoacoustic oscillation.
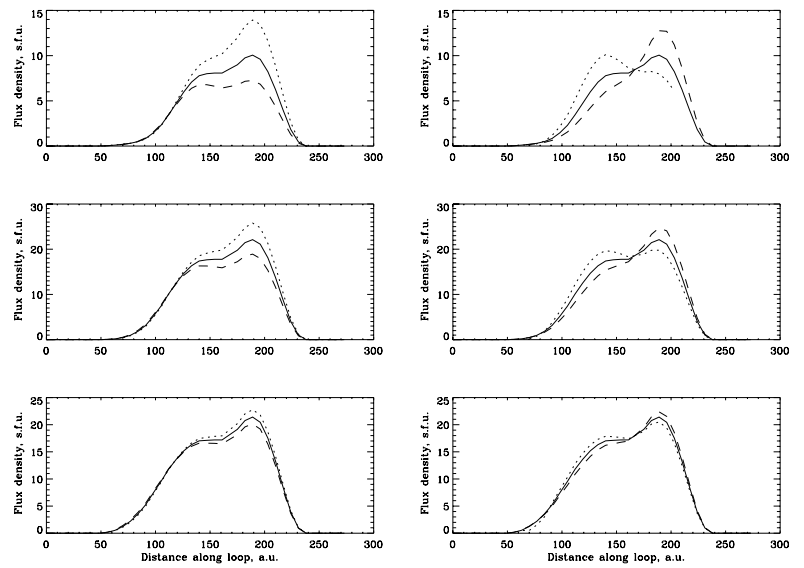

Fig. 6. The distribution of the microwave emission along the projection of a coronal loop in the global slow magnetoacoustic mode (left column) and in the second harmonics (right column) at different frequencies. The upper row corresponds to $9.4 \mathrm{GHz}$, the middle row to $17 \mathrm{GHz}$, and the bottom row to $34 \mathrm{GHz}$. The line-of-sight is assumed to be in the loop plane and to be inclined to the right with angle of $60^{\circ}$ to the horizon. The units of the horizontal axis are arbitrary and correspond to the units shown in Fig. 5. The plasma density is $8 \times$ $10^{10} \mathrm{~cm}^{-3}$, the temperature is $10^{7} \mathrm{~K}$, and the magnetic field strength is $100 \mathrm{G}$. All plasma parameters, the magnetic field strength, and the non-thermal electron density are taken to be uniform along the loop. The solid lines correspond to the equilibrium density. The dotted and dashed curves correspond to the maximum and the minimum phases (the upper and the lower rows of Fig. 1, respectively) of the slow magnetoacoustic modes with the relative amplitude of $10 \%$.

the solid curves in Fig. 6. The curves were calculated with the numerical code discussed in Sect. 2. The segments of the loop, which have the enhanced column depth, contribute to the elephant's back in the profile, and the the segments with the magnetic field perpendicular to the LOS contribute to the elephant's head.

When the loop experiences slow magnetoacoustic oscillations, the plasma density along the loop axis varies in time (see Fig. 1), while the magnetic field remains practically unperturbed by this mode. The dotted and dashed curves in Fig. 6 show the modification of the microwave image of the loop for the given LOS at different phases of oscillations. 

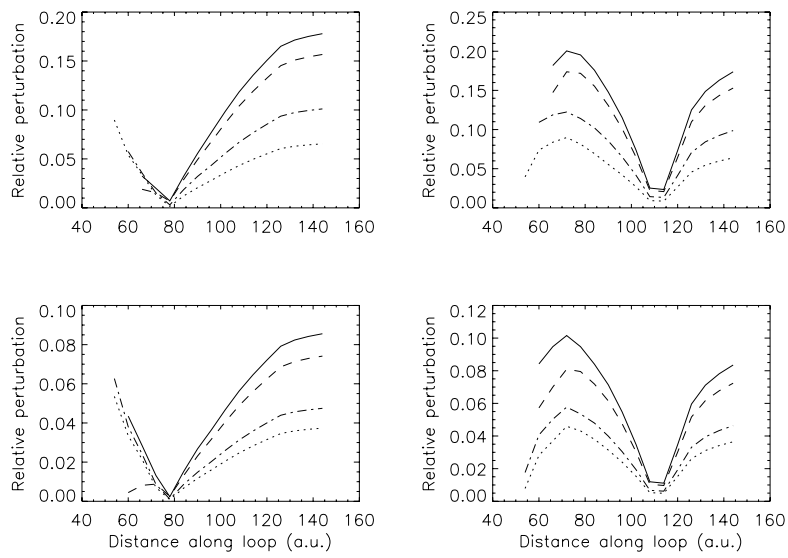

Fig. 7. The observed relative amplitude of the microwave emission along the projection of a coronal loop in the global slow magnetoacoustic mode (left column) and in the second harmonics (right column) at different frequencies for different physical conditions in the loop. The upper row corresponds to $9.4 \mathrm{GHz}$, and the bottom row to $17 \mathrm{GHz}$. The solid curves correspond to the case with the LOS angle of $60^{\circ}$, the plasma density of $8 \times 10^{10} \mathrm{~cm}^{-3}$, the temperature of $10^{7} \mathrm{~K}$, the magnetic field strength of $100 \mathrm{G}$, and the non-thermal electron energy spectral index $\delta=3$. All plasma parameters and the density of the non-thermal electrons are taken to be uniform along the loop. The other curves correspond to the same combination of parameters, but with the magnetic field of $200 \mathrm{G}$ (the dotted curves), with the spectral index equals to 5 (the dashed curves), and the plasma density of $5 \times$ $10^{10} \mathrm{~cm}^{-3}$ (the dot-dashed curves).

The observed modulation depth varies along the loop and is stronger at lower observation frequencies, which is consistent with Fig. 4. Figure 6 demonstrates that different slow modes have quite distinct observational signatures in the loop's microwave image.

From the observational point of view, a more convenient way to visualise the modal structure is to use the observed relative perturbation,

$A_{\mathrm{obs}}=\frac{I_{f}^{\max }-I_{f}^{\min }}{I_{f}^{\max }+I_{f}^{\min }}$,

where $I_{f}^{\max }$ and $I_{f}^{\mathrm{min}}$ are the maximum and the minimum values of the modulated emission $I_{f}$ observed at the frequency $f$, cf. with the modulation factor given by Eq. (4). Figure 7 shows the structure of the relative perturbation (5) along a loop in the global slow magnetoacoustic mode and in its second harmonics for different parameters, at two frequencies, $9.4 \mathrm{GHz}$ and $17 \mathrm{GHz}$, for one particular view angle. According to Fig. 3 these frequencies correspond to the strongest gyrosynchrotron signal. In the calculation of the relative perturbation, we neglected the loop segments where the signal was lower than a certain threshold, which allowed us to avoid the calculation of the ratio of too small quantities. According to the figure, the node of the density perturbation in the global mode is resolved well in all cases studied. The same is true for one of the nodes of the second harmonics, while the second node is not well-resolved. This makes it difficult to distinguish between the global modes and the second harmonics observationally when based only on analysis of the spatial structure of the mode. Also, it is obvious that the position and the depth of the density nodes in the modulated emission along the projected loop depends upon the view angle. However, detection of a node in data with spatial resolution would be a strong indication of the slow magnetoacoustic standing wave in the loop.

\section{Conclusions}

We theoretically modelled the modulation of a microwave emission generated in a flaring loop by long period slow magnetoacoustic waves. We summarise our findings as follows:

- Slow magnetoacoustic oscillations, which are confidently detected in coronal loops in the EUV band, can be responsible for long period (ranging from a few tens of seconds to several min) modulation of the microwave emission generated in flaring loops.

- At all microwave frequencies, modulation of the emission is found to be in anti-phase with the density perturbation induced by the slow oscillations.

- The observed modulation depth can be up to an order of magnitude higher than the modulation of the plasma density in the slow wave. The modulation is more pronounced in the low frequency part of the microwave spectrum. For typical loop parameters, the most suitable popular microwave channels for the detection of slow magnetoacoustic modes are $9.4 \mathrm{GHz}$ and $17 \mathrm{GHz}$.

- Slow modes can have clear and distinct signatures in the loop's microwave images. The presence of a node of the relative perturbation of the emission indicates the slow magnetoacoustic nature of the microwave emission modulation, provided its period is consistent with the estimation given by Eq. (1) or its second harmonics. The mode interpretation should be based upon analysis of both the spatial structure of the emission modulation and the modulation period.

Thus, slow magnetoacoustic waves can be responsible for long period variations of the microwave emission of coronal loops. We would like to point out that standing slow magnetoacoustic modes are usually observed to be of low quality. For example, the decay time of SUMER oscillations is found to be just a few oscillations periods (Wang et al. 2003), and the oscillations associated with the flare-generated second slow harmonics (Nakariakov et al. 2004) occur in trains with only several periods. Moreover, the resonant periods of the standing slow oscillations depend upon the loop length and the temperature, (Eq. (1)) and both parameters, especially the temperature, are known to vary during flares. Consequently, the search for slow oscillations in the microwave data should be based upon the use of a dynamical spectral tool or wavelet analysis. Another important restriction on the observability of slow modes is connected with the requirement that the duration of particle acceleration process (the duration of the flare) should be longer than several periods of the oscillation. Practically, microwave light curves can contain the superposition of the oscillations produced by slow modes and other dynamical phenomena associated with the electron acceleration. In addition, during a flare, the background plasma density can vary significantly, up to one order of magnitude. According to the top-left panel of 
Fig. 4, the modulation of the gyrosynchrotron emission is very sensitive to variation in the background plasma density, which should be taken into account in interpretations of observations. In particular, decrease in the density in the decay phase of a flare will reduce the modulation factor, causing damping of the observed modulation. The results presented above demonstrate that slow modes are detectable in the microwave band with modern radioheliographs, providing us with an interesting data analysis challenge.

Acknowledgements. The authors are grateful to the referee, Dr T. J. Wang. The work was partly supported by the RFBR grants No. 04-02-39029, 04-02-16753 and by the Royal Society British-Russian Research Collaboration grant. V.M.N. acknowledges the support of a Royal Society Leverhulme Trust Senior Research Fellowship and the EPSRC grant GR/R77940/1.

\section{References}

Asai, A., Shimojo, M., Isobe, H., et al. 2001, ApJ, 562, L103

Aschwanden, M. J. 2004, Physics of the Solar Corona (Berlin: Springer Praxis Books)

Baranov, N. V., \& Tsvetkov, L. I. 1994, Astron. Lett., 20, 327

Berghmans, D., \& Clette, F. 1999, Sol. Phys., 186, 207

DeForest, C. E., \& Gurman, J. B. 1998, ApJ, 501, L217

De Moortel, I., Hood, A. W., Ireland, J., \& Walsh, R. W. 2002, Sol. Phys., 209, 61
Fleishman, G. D., \& Melnikov, V. F. 2003, ApJ, 584, 1071

Gary, D. E., Linsky, J. L., \& Dulk, G. A. 1982, ApJ, 263, L79

Gundyrev, A. G., \& Razin, V. A. 1995, Astron. \& Astrophys. Trans., 6, 229

Khodachenko, M. L., Zaitsev, V. V., Kislyakov, A. G., Rucker, H. O., \& Urpo, S. 2005, A\&A, 433, 691

Melnikov, V. F., Reznikova, V. E., Shibasaki, K., \& Nakariakov, V. M. 2005a, A\&A, 439, 727

Melnikov, V. F., Gary, D. E., \& Nita, G. M. 2005b, ApJ, in press

Mendoza-Briceño, C. A., Erdélyi, R., \& Sigalotti, L. D. 2004, ApJ, 605, 493

Nakariakov, V. M., Melnikov, V. F., \& Reznikova, V. E. 2003, A\&A, 412, L7

Nakariakov, V. M., Tsiklauri, D., Kelly, A., Arber, T. D., \& Aschwanden, M. J. 2004, A\&A, 414, L25

Nakariakov, V. M., \& Verwichte, E. 2005, Living Rev. Solar Phys., 3,1

URL: http://www . livingreviews .org/lrsp-2005-3

Nakariakov, V. M., Verwichte, E., Berghmans, D., \& Robbrecht, E. 2000, A\&A, 362, 1151

Ofman, L., Nakariakov, V. M., \& DeForest, C. E. 1999 ApJ, 514, 441

Ofman, L., \& Wang, T. J. 2002, ApJ, 580, L85

Ramaty, R. 1969, ApJ, 158, 753

Razin, V. A. 1960, Izv. VUZov Radiofizika, 3, 584

Roberts, B., Edwin, P. M., \& Benz, A. O. 1984, ApJ, 279, 857

Wang, T. J., Solanki, S. K., Curdt, W., Innes, D. E., \& Dammasch, I. E. 2002, ApJ, 574, L101

Wang, T. J., Solanki, S. K., Curdt, W., et al. 2003, A\&A, 406, 1105 